\title{
Cuerpo, sujeto y saber en un Espacio de Formación Integral
}

\author{
Paola Dogliotti \\ paoladogliottimoro@gmail.com
}

Paula Malán

paulamalan@gmail.com

Juan Muiño

juan_1331@hotmail.com

\author{
Giannina Silva \\ gianninarss@gmail.com \\ Universidad de la República, \\ Uruguay
}

Cultura(s) en clave de extensión universitaria / Intervenciones
RECEPCIÓN: 02/04/19

ACEPTACIÓN FINAL: 30/05/19

\section{Resumen}

El trabajo presenta los principales avances analíticos y problematizaciones llevadas adelante por el Espacio de Formación Integral "Cuerpo y Educación en Malvín Norte" desarrolladas durante el año 2018 en el barrio Malvín Norte, en la ciudad de Montevideo, Uruguay. El análisis gira en torno a cuatro categorías centrales: cuerpo, enseñanza, educación y sujeto. Se parte de un trabajo de inmersión territorial que permitió construir una serie de registros de observación participante, entrevistas y documentos institucionales. Se problematiza sobre la tensión entre la producción y la reproducción de prácticas educativas, y en especial sobre el lugar del cuerpo, el saber y el sujeto en la educación y en la enseñanza, mostrando los principales ejes y aristas según la problemática específica seleccionada por cada una de las cuatro instituciones socioeducativas que formaron parte de la experiencia de este Espacio.

Palabras clave: cuerpo, educación, enseñanza, Malvín Norte, Espacio de Formación Integral.
Body, subject and knowledge in an Integral Training Space

\section{Abstract}

The work introduces the main analytical advances and questions carried out by the Integral Training Space "Body and Education in Malvín Norte" during the year 2018. This space took place in the neighborhood Malvín Norte, in the city of Montevideo, Uruguay. The analysis was based on four central categories: body, teaching, education and subject. The immersion in the territory allowed the construction of a series of participant observations, interviews and institutional documents. The work inquires into the tension between reproduction and production of educational practices, and especially about the place of the body, the knowledge and the subject, in education and in teaching, showing the main axes and edges of the specific problem selected for each of the four socioeducational institutions that were part of the Integral Training Space experience.

Keywords: body, education, teaching, Malvín Norte, Integral Training Space.
Corpo, sujeito e conhecimento no Espaço de Formação Integral

\section{Resumo}

O trabalho apresenta os principais avanços e problematizações realizadas pelo Espaço de Capacitação Integral (EFI) "Corpo e Educação no Malvín Norte", desenvolvido no ano de 2018, no bairro de Malvín Norte, na cidade de Montevideo, Uruguai. A análise gira em torno de quatro categorias centrais: corpo, ensino, educação e sujeito. É baseado em um trabalho de imersão territorial que permitiu a construção de uma série de registros de observação participante, entrevistas e documentos institucionais. O trabalho problematiza a tensão entre a reprodução e a produção de práticas educativas e, principalmente, o lugar do corpo, do saber e do sujeito, na educação e no ensino, mostrando os principais eixos e arestas do problema específico selecionado para cada uma das quatro instituições socioeducativas que faziam parte da experiência da EFI.

Palavras-chave: corpo, educação, ensino, Malvín Norte, Espaço de Capacitação Integral.

Para citación de este artículo: Dogliotti, P.; Malán, P.; Muiño, J. y Silva, G. (2019). Cuerpo, sujeto y saber en un Espacio de Formación Integral. +E: Revista de Extensión Universitaria, 9(10), 124-136. doi: 10.14409/extension.v9i10.Ene-Jun.8295. 


\section{Introducción}

El trabajo se propone presentar un análisis y sistematización del Espacio de Formación Integral (EFI) titulado "Cuerpo y Educación en Malvín Norte" desarrollado en el año 2018. En la Universidad de la República de Uruguay (UdelaR).

"Ios EFI son ámbitos para la promoción de prácticas integrales en la Universidad, favoreciendo la articulación de enseñanza, extensión e investigación en el proceso formativo de los estudiantes, promoviendo el pensamiento crítico y propositivo, y la autonomía de los sujetos involucrados. Las prácticas integrales promueven la iniciación al trabajo en grupo desde una perspectiva interdisciplinaria, donde se puedan vincular distintos servicios y áreas del conocimiento, reunidos por una misma temática, un territorio o problema. De este modo, los EFI son dispositivos flexibles, que se conforman a partir de múltiples experiencias educativas en diálogo con la sociedad —prácticas, cursos, talleres, pasantías, proyectos de extensión y/o investigación- asumiendo diferentes formas de reconocimiento curricular según las características de cada servicio." (UdelaR, 2010:9-10)

Para el caso de nuestro EFI, estudiantes y docentes de tres institutos de formación ${ }^{1}$ se insertan a diversas experiencias territoriales llevadas adelante por instituciones socioeducativas (escuelas, liceos y organizaciones de la sociedad civil), al grupo de investigación "Políticas Educativas, Cuerpo y Curriculum" ${ }^{2}$ y a la línea de investigación "Políticas Educativas, Cuerpo y Enseñanza" ${ }^{3}$. En la articulación entre el objeto de estudio de los grupos y líneas de investigación, la problemática de cada una de las instituciones y los principales nudos conceptuales abordados en algunos cursos de las carreras respectivas, se estructura el EFI y se define la propuesta concreta a llevarse adelante por cada subgrupo de trabajo en territorio.

El problema central que el EFI se plantea es el lugar del cuerpo en la educación y en la enseñanza y, en esta relación, del sujeto de la educación. Desde allí se busca potenciar el trabajo realizado por las diferentes instituciones, favorecer la reflexión, sistematización y colectivización de sus propuestas y profundizar teóricamente en el análisis y abordaje de las problemáticas educativas y de enseñanza desde la especificidad de lo corporal, contribuyendo con los actores institucionales a la producción de conocimiento sobre esta temática.

El trabajo presenta los principales avances analíticos y problematizaciones llevadas adelante con los equipos de trabajo de las cuatro instituciones seleccionadas en el año 2018 (el Centro Juvenil Lamistá, la escuela común № 268, la escuela de tiempo completo $N^{\circ} 267$ y el Liceo $N^{\circ} 42$ ) respecto de cuatro categorías centrales: cuerpo, enseñanza, educación y sujeto.

En coordinación con cada uno de los equipos institucionales se desarrollan a continuación los problemas de intervención abordados en cada uno a lo largo del año 2018, luego

\footnotetext{
1) Participan de este EFI estudiantes de la Licenciatura en Educación Física del Instituto superior de Educación Física (ISEF), de la Licenciatura en Educación de la Facultad de Humanidades y Ciencias de la Educación (FHCE) y de la carrera de Educador Social del Consejo de Formación en Educación (CFE) de la Administración Nacional de Educación Pública (ANEP).

2) Grupo inscripto en el Departamento de Educación Física y Prácticas Corporales de ISEF.

3) Línea inserta en el Departamento de Enseñanza y Aprendizaje del Instituto de Educación de la FHCE.
} 
de haber realizado durante 2017 y el segundo semestre de 2016 un acercamiento general a ocho instituciones socioeducativas y a la red educativa de la zona. ${ }^{4}$

1. En el Centro Juvenil Lamistá se llevó a cabo el análisis/sistematización de un espacio de taller semanal denominado "Expresión Artística" a partir de una observación participante de este y de una intervención mensual en la reunión del equipo del proyecto.

2. En la escuela común № 268 se sistematizó de un espacio de "Talleres Multigrado" efectuados en forma quincenal por parte de la comunidad educativa de la escuela, en donde se rompe el formato del "grupo-clase" y se integran niños y niñas de todos los grados a cada uno de los talleres.

3. En la escuela de tiempo completo $N^{\circ} 267$ se realizó una intervención en el espacio semanal de reunión del equipo docente a partir de registros e intervenciones que colaboraron en la elaboración del proyecto de centro5 y en el análisis del cambio del formato de escuela común a escuela de tiempo completo ocurrido en el año 2018.

4. En el Liceo № 42 se hizo una intervención de potenciación del espacio de coordinación semanal de los profesores ayudando al armado de un proyecto de centro y al mejoramiento de la imagen institucional del Liceo.

Cabe destacar que el proceso de definición del problema de intervención se desarrolló con el diálogo y reflexión conjunta entre los actores territoriales y los universitarios y se fue transformando y definiendo a lo largo del año 2018. Podríamos decir que se trazó una primera "imagen-objetivo" de lo que pretendíamos realizar en los primeros meses del año (febrero a abril) y luego se fue redefiniendo, ajustando, en función del recorrido desplegado en cada espacio institucional-territorial.

Por medio de la presencia quincenal en cada una de estas instituciones se recolectó una serie de registros de observación participante de diversos espacios (talleres, reuniones de equipo, espacios de recreo, de bienvenida, entre los centrales), entrevistas, fotografías, documentos institucionales (proyectos de centro, evaluaciones diagnósticas), entre los principales instrumentos de recolección de información.

\section{Algunas grillas conceptuales}

El marco teórico del EFI parte de la distinción entre educación y enseñanza, en tanto la primera remite al gobierno de las poblaciones y al disciplinamiento de los cuerpos (Foucault, 1992) y la segunda se centra en la trasmisión y producción del saber-conocimiento (Behares, 2004). Se muestran las principales tensiones y articulaciones de estas dos dimensiones en las cuatro experiencias analizadas y las discusiones generadas por sus colectivos docentes

4) La Red Educativa de Malvín Norte surge en el año 1999 y se ha mantenido como una instancia de coordinación de actividades entre las instituciones educativas, sociales y de salud vinculadas a niños y adolescentes del barrio. Actualmente se viene trabajando en la necesidad de articulación entre las diversas instituciones de la zona con el fin de atender las dificultades para la continuidad educativa de los estudiantes que egresan de primaria e ingresan a educación media así como los problemas de desvinculación estudiantil de primaria y educación media.

5) Proyecto de centro es como se denomina en el Uruguay a la planificación anual institucional, transversal a todas las acciones y actividades de la institución, que tiene como responsable a la dirección institucional pero que generalmente se elabora a partir de la participación del colectivo docente. 
en torno a ellas. ¿De qué forma se vinculan la educación y la enseñanza? Desde esta distinción se trabaja sobre el lugar del cuerpo en la educación y en la enseñanza.

El enfoque teórico-metodológico es sobre el análisis discursivo afectado fundamentalmente por el análisis de discurso de Pêcheux (1988), quien concibe al discurso como estructura y acontecimiento. La estructura, del orden del lenguaje, es organizadora de sentido, mientras que el acontecimiento es del orden de la inestabilidad semántica. De este modo, en todo acto enunciativo se entrecruzan fórmulas de apariencia lógica y estable y fórmulas equívocas. Esto permite pensar la educación, la enseñanza y el cuerpo como dimensiones de aquella estructura que se organiza en torno a la reproducción-transmisión de conocimientos históricamente configurados pero que incluye el acontecimiento: la apertura a la singularidad del sujeto escindido, la posibilidad de falla de la estructura, la entrada de un saber en falta. Behares (2010) presenta este saber en falta en el centro de la razón de ser de la enseñanza, aquello que convoca al deseo del enseñante y por lo cual enseña. Nuevamente Behares (2007) expresa, a partir de una afectación psicoanalítica, que este saber es de orden pulsional. Así, el cuerpo como objeto de educación/enseñanza en el aula de las cuatro instituciones seleccionadas está expuesto a estos dos movimientos que conforman la dialéctica del saber: la estructura (redes de sentido históricamente conformadas y representables, el conocimiento sobre el cuerpo) y el acontecimiento (el lugar de la falta, del equívoco) (Dogliott, 2007), del orden del saber del cuerpo. Es en esta tensión que pretendemos trabajar y problematizar en cada una de las experiencias seleccionadas sobre el lugar del cuerpo y del sujeto en la educación y la enseñanza.

Luego del desarrollo anterior, consideramos que el cuerpo no puede ser entendido ni analizado fuera de una trama discursiva. Si bien en el establecimiento de los sistemas educativos modernos este se ha configurado en forma hegemónica desde lo anátomo-fisiológico, remitiéndolo, por medio de saberes que provienen de manera primordial de la biología y de la medicina, a un conjunto de aparatos y órganos, nos parece central ubicarlo como efecto de las materialidades lingüísticas histórica y socialmente conformadas. "Los discursos sobre el cuerpo son históricamente configurados en una trama de sentidos pero es la materialidad de la palabra en tanto significante la que permite abrir a la alteridad y al cambio" (Dogliotti, 2007:132).

Lo lúdico, lo expresivo, lo creativo y lo artístico y su relación con lo corporal cobrarán especial atención en el análisis de las experiencias ¿Qué espacios de creación se promueven desde las diversas propuestas, qué lugar tiene el cuerpo y cómo se configura en cada una de ellas?

Dentro de la problemática central del lugar del cuerpo en la educación, se aborda el tema de qué discursos en torno al sujeto de la educación están presentes en las cuatro experiencias ¿Cómo se piensa el lugar del sujeto de la educación en el territorio? Desde perspectivas teóricas que reflexionan sobre este tema, entre las que se destaca la de Martinis (2006) se analizan las tensiones y solapamientos entre el discurso del sujeto carente y el del sujeto de la posibilidad en la educación.

El análisis al que refiere este artículo representa un primer producto del trabajo de reflexión y problematización que nuestro EFI lleva adelante en las cuatro instituciones desde el año 2017.

El artículo se estructura en cuatro apartados, en cada uno de ellos se desarrolla la experiencia en territorio de las instituciones y se plantean diálogos con el marco teórico. Este diálogo en ningún momento pretende encasillar o afirmar la teoría a partir de un caso sino mostrar de qué modo los casos permiten poner en tensión la teoría, mostrar sus faltas. Las 
preguntas que se proponen a lo largo del análisis procuran interpelar al lector y promover nuevos acercamientos a la experiencia en territorio desde la teoría; ellas formaron parte del trabajo con los actores institucionales y universitarios a lo largo del proceso del EFI. Se cierra con una serie de consideraciones finales que intentan articular los principales cuestionamientos desarrollados en cada uno de los apartados.

\section{Las experiencias en el territorio}

\section{a) Taller de Expresión Artística Centro Juvenil Lamistá}

Para el caso de este Centro Juvenil, el trabajo de análisis y discusión conceptual se centró en distintos insumos que se fueron produciendo a través del registro del taller de Expresión Artística y del correspondiente a las dinámicas impulsadas por el conjunto de estudiantes universitarios en la reunión de equipo del Centro. En este sentido, el análisis y la problematización de la práctica educativa de esta institución se mueven entre una instancia general y una específica. La instancia general intenta recuperar algunos aspectos globales de la propuesta educativa del Centro (esto es, más allá de espacios concretos, como, por ejemplo, el propio taller de expresión artística), relacionados con su rutinización, sus disposiciones temporales y espaciales y sus objetivos, entre otros aspectos. Para esto, en algunas de las primeras dinámicas propuestas al equipo de trabajo en los espacios de reunión se impulsaron dinámicas que permitieron recolectar datos para la reconstrucción y discusión del funcionamiento general y cotidiano de la institución, que se acompañaron de una serie de registros a partir de observaciones participantes de parte del funcionamiento diario. La instancia específica recupera el funcionamiento del taller de Expresión Artística mediante su sistemático seguimiento y registro, a través también de la observación participante, en cada una de sus ediciones.

Por un lado, consecuentemente con el punto de vista teórico del que parte nuestro proyecto, el análisis pone su foco en la interpretación de la práctica educativa, en ocasión del cuerpo, en su funcionamiento oscilante entre aquello que tiende a la reproducción y aquello que la agujerea por todas partes, imposibilitando su saturación y cierre sobre sí misma. Tomando como referencia los aportes de Agamben, se trabaja con juego y rito como categorías analíticas que aportan herramientas conceptuales especialmente fértiles para la intelección de estos funcionamientos de la práctica con relación a su eje temporal. Al respecto:

"podemos conjeturar una relación al mismo tiempo de correspondencia y de oposición entre juego y rito, en el sentido de que ambos mantienen una relación con el calendario y con el tiempo, pero que dicha relación es inversa en cada caso: el rito fija y estructura el calendario, el juego en cambio, aun cuando todavía no sepamos cómo ni porqué, lo altera y lo destruye". (2003:98-99)

La sistematización de los registros fue arrojando que, en su cotidianidad, el Centro Juvenil, como toda institución educativa, insiste en la rutinización de determinadas dinámicas que se disponen temporalmente de forma ordenada, organizando la práctica y el tránsito de los sujetos por ella, a modo de ritualización. Dentro de estas, se destaca "la ronda", espacio que abre y cierra la jornada del Centro, cuando, por un lado, se organizan las actividades 
del día y, por otro, se repasa y evalúa lo realizado en la jornada. Con todo, es importante destacar que, si bien estos momentos ritualizantes se configuran como espacios en los que el ordenamiento de los cuerpos tiende a su reproducción, no se debe perder de vista, con Dogliotti (2007), que se contiene allí el lugar para la irrupción del acontecimiento, de aquello del cuerpo que se resiste a la homogenización. De manera que, más allá de modos de estar y de percibir que se tornan habituales para el ordenamiento y disciplinamiento del cuerpo (Foucault, 2015; 1992), se encuentra también en la propia ritualización el fondo sobre el cual emerge lo contingente que suspende la reproducción.

Asimismo, se pudo observar que la propuesta del Centro Juvenil prevé tiempos libres en los que los adolescentes funcionan con autonomía no obstante los direccionamientos institucionales. Apoyadas en las observaciones, las primeras reflexiones analíticas a propósito de estas circunstancias muestran que se establecen allí momentos en los que predominan el juego, los chistes, bailes y cantos, donde prima la espontaneidad, encontrándose elementos para pensar que algo se renueva. En consonancia, el taller de expresión artística, de acuerdo con los registros, se establece también, desde los objetivos que se proponen, como un espacio en el que se habilitan diferentes formas de poner el cuerpo y habitar el espacio dando lugar a lo nuevo. Sin embargo, sobre el funcionamiento de los mencionados tiempos libres con relación a la oposición juego/rito, es importante ingresar algunos matices interpretativos que resultan, en parte, de las discusiones que se desarrollaron en las reuniones con el equipo del Centro Juvenil en el proceso de escritura y reflexión desarrollado. Un matiz, para nada menor, refiere a que estos espacios, en los que parece primar el juego, pueden ser también objeto de calendarización y rutinización en el marco de la propuesta del Centro y contener los modos de la reproducción de la estructura. Con él, el análisis y su problematización con el equipo del Centro se colocan frente a un funcionamiento más refinado de la oposición entre juego y rito, y es que "todo juego (...) contiene una parte de rito y todo rito una parte de juego, lo que con frecuencia hace difícil la distinción entre uno y otro" (Agamben, 2003:107). Igualmente, en sus relaciones con la estructura y el acontecimiento: "mientras que el rito (...) transforma los acontecimientos en estructuras, el juego transforma las estructuras en acontecimientos" (Lèvi-Strauss en Agamben:106).

Con relación a esto, los educadores de la institución manifiestan que aquello que acontece de forma imprevista es tenido en cuenta a la hora de planificar la práctica, reservando un lugar particular para lo contingente que desestabiliza lo previsto. Se encuentra allí una tensión entre la preocupación por sostener lo cotidiano, lo que "tiene que ser", frente a lo no planificado, que constantemente emerge, a modo de absorción del acontecimiento en la estructura y resolución de las contradicciones entre pasado y presente. Por eso la preocupación por reservar un lugar para lo imprevisto frente a la rutinización tendiente a la reproducción, de manera que los acontecimientos que desmigajan la estructura a partir del juego puedan ser contemplados como parte de la práctica educativa. Este funcionamiento, propio de lo educativo, se configura, acompañado por el análisis y la problematización con

6) Es importante advertir que los términos estructura y acontecimiento utilizados aquí (trabajados por Agamben a partir de Lèvi-Strauss) no son intercambiables por los introducidos con Pêcheux (1988) en el inicio, que privilegian al sujeto en la estructura con relación al discurso. Sin embargo, desde ambas entradas, grosso modo, el análisis apunta a observar cómo lo educativo funciona en una tensión irreductible. 
el equipo, como un elemento que atraviesa y tensiona toda la propuesta del Centro Juvenil, tanto en su generalidad como en sus espacios específicos, entre los que se incluye el Taller de Expresión Corporal.

Con todo, este fragmento del análisis no hace justicia al trabajo de problematización desarrollado con el Centro Juvenil y funciona, antes que todo, a modo de ejemplo. Por razones evidentes, se dejaron de lado los desarrollos del análisis desprendidos de estas categorías, así como otras categorías analíticas que son centrales en el trabajo, a saber: enseñanza, saber, técnica y control.

\section{b) Talleres Multigrado escuela $N^{\circ} 268$}

Para llevar adelante el trabajo de sistematización de los Talleres Multigrado en la escuela № 268 nos involucramos desde un lugar de observadores participantes en ellos. Realizamos entrevistas a los referentes institucionales (equipo de dirección, maestras, auxiliares) y buscamos diversas instancias de diálogo con los maestros, con las auxiliares y con el equipo de dirección para compartir reflexiones en torno al cuerpo y la educación y acercar elementos de la teoría que resulten significativos para hacernos preguntas que nos ayuden a repensar las prácticas educativas. ${ }^{7}$

La propuesta de talleres en la escuela opera como ruptura del modelo pedagógico tradicional, respecto del formato "grupo-clase", y permite que niños de diferentes grupos y grados compartan este espacio en función de sus intereses, a la vez que comparten con otros docentes. Por otra parte, esta propuesta posibilita desviar el foco hacia otros saberes que no son los formales y que mucho tienen que ver con el saber artístico, el saber popular, el saber-hacer, y con las dimensiones expresivas, comunicativas y creativas. Dentro de la oferta de talleres propuesta por los docentes en el año 2018 se encuentran: Murga, Arte, Cocina, Tejido, Plástica y Origami, Construcción de juegos y juguetes, Manualidades, y Construcción de materiales para la Educación Física. La propuesta de los talleres, dentro del proyecto de centro es considerada por el colectivo docente como una "propuesta de convivencia" en la que está integrada toda la escuela, ya que otorga la posibilidad del trabajo en duplas entre maestros, la creación de vínculos entre niños de diferentes edades, que hermanos compartan el mismo espacio, que las familias puedan colaborar de diferentes maneras (compartiendo una receta, ayudando en la construcción de un material, etc.) y que las auxiliares se integren al trabajo pedagógico. La "Fiesta de la Escuela", cuando esta abre sus puertas a la comunidad, tiene lugar con el cierre y la muestra de los talleres, donde cada uno presenta el producto del trabajo realizado durante todo el año. ${ }^{8}$

7) Cabe destacar que en esta institución, a diferencia de las restantes, no hubo posibilidad, más que en el mes de febrero, de insertarnos en las reuniones de equipo docente, ya que esta escuela no tiene espacios de reunión para tal efecto de no ser en los tres días administrativos del mes de febrero. Frente a esta dificultad, se trató de involucrar a las maestras, personal de servicio y equipo de dirección mediante encuentros esporádicos en forma individual, en los espacios de recreo, o en otras instancias a lo largo del espacio escolar.

8) Desde el equipo universitario se logró para este evento presentar en forma de banner el Espacio de Convivencia para mostrar la experiencia de sistematización de los talleres por parte del EFI. Este fue uno de los productos concretos que logró el EFI durante 2018, además del producto de sistematización compartido con el equipo docente en el espacio de reuniones del mes de febrero de 2019. 
El foco de análisis estuvo puesto en la pregunta por el lugar del cuerpo en esta instancia educativa, partiendo de la afirmación de Milstein y Mendes de que "el trabajo pedagógico con alumnos implica siempre un trabajo con y en el cuerpo -más o menos explícito-y que este trabajo es la base y la condición de los demás aprendizajes" (1999:17).

En primer lugar, desde los registros de observación participante en los talleres surge la tensión por la ritualización/inscripción del cuerpo en una trama discursiva. Los talleres rompen con la disposición homogénea de los niños frente al pizarrón y con la noción de un tiempo individual de la tarea, para proponer una disposición heterogénea en pequeños grupos - conformados por niños de diferentes grados-, donde no hay un frente, pues el docente o los docentes circulan por los pequeños grupos acompañando la propuesta, y el tiempo se vuelve grupal. Por lo tanto, hay un corrimiento/desplazamiento de ciertas lógicas discursivas ya instaladas y naturalizadas en la cultura escolar, donde los cuerpos se inscriben de un modo espacial y temporal particular hacia otras lógicas discursivas que posibilitan una nueva forma de relación de los cuerpos. Las nociones de disciplinamiento, autocontrol, inmovilidad, eficacia, individualización se ven tensionadas (Foucault, 1992; 2015). El cambio en la disposición temporal y espacial de los cuerpos habilita la circulación de otros discursos, de otros significantes: el encuentro con otros cuerpos en otro tiempo y en otro espacio, que es grupal, coloca en el centro de la trama discursiva a la expresión, la comunicación y la creación, a partir del intercambio y el diálogo con los compañeros — de mesa cuando la hay, de banco, o simplemente con los compañeros que se agrupan en un espacio en común-y el docente que retroalimenta circulando por los pequeños grupos. Entonces nos preguntamos: ¿qué habilita esta nueva disposición de los cuerpos en el espacio y en el tiempo en cuanto a la subjetividad, a lo social y al saber? ¿De qué modo este espacio y tiempo común amplía las posibilidades de encuentro con el saber en la medida en que la relación de los otros con el saber puesto en juego afecta/interpela la relación individual con este? ¿De qué manera el intercambio entre niños de diferentes grados predispone al acercamiento con el saber? ¿De qué forma este múltiple acercamiento enriquece la propuesta educativa del docente?

En segundo lugar, lo corporal se observa en la dimensión de lo sensible y lo cotidiano. Los sentidos están presentes en todos los talleres, por ejemplo, la manipulación de la masa, el aroma y el gusto de los alimentos en el taller de Cocina; la combinación de texturas y colores en los talleres de Arte y Manualidades; los ritmos y los compases en el taller de Murga. El hecho de convocar la percepción trae consigo el acercamiento al mundo cotidiano, la relación de lo nuevo con lo ya conocido -al preparar una nueva receta se pregunta qué se ha cocinado en casa que lleve este ingrediente, cuando se mezclan los colores se busca observar el paisaje que rodea el barrio, cuando se elabora la letra de la murga se habla sobre acontecimientos vividos-, un intercambio cultural entre la escuela, la familia y la comunidad, una relación de los contenidos formales del programa escolar con los saberes que circulan en el medio social, para dar lugar a un nuevo saber, un saber del cuerpo que convoca e interpela la historia y la memoria subjetiva y social de los sujetos. Aquí nos preguntamos de qué manera la inscripción de lo sensible con relación a lo cotidiano habilita el deseo de saber del sujeto donde lo corporal de orden pulsional está presente. Los discursos circulan, los significantes insisten en inscribirse en el cuerpo de los sujetos, pero los cuerpos resisten y la inscripción nunca es plena, siempre hay algo del orden pulsional del sujeto que se manifiesta en las fallas de ese ritual de inscripción, y el deseo del sujeto 
no cesa de no inscribirse. "Para el sujeto la falta de gozar y la falta de saber son, en realidad, la misma falta, la falta en el orden pulsional. (...) O lo que es lo mismo: todo saber (y obviamente su falta y su deseo) es de origen pulsional" (Behares, 2007:9). Consideramos que la dimensión de lo sensible y lo cotidiano interpela la historia y la memoria del sujeto en su relación con el saber del mundo y así convoca la cadena significante y su vacío mientras posibilita la resignificación.

Por último, nos detenemos a pensar acerca del saber técnico que se pone en juego en la creación de un producto final, que es el objetivo de la propuesta de los talleres. Hay un encuentro con el hacer manual, con la elaboración de un objeto material, con la creación de un número artístico en donde el cuerpo se mueve, gesticula, habla, canta, se expresa, se comunica, crea. Aparece aquí la relación con una técnica o una práctica corporal. Y nos preguntamos: ¿qué relación guarda este discurso técnico con su origen? ¿Se constituye en una secuencia de acciones prácticas que han sido desprendidas del discurso que le dio origen? ¿Es este recurso un fragmento de saber alienado, "reificado", en palabras de Chevallard (1991)? ¿De qué modo el docente se las ingenia para restituir a la técnica su relación con el saber? Siguiendo las ideas de Alonso (2007), ¿cómo es posible posicionar a la técnica respecto de la formación corporal del sujeto en vez de ser un medio de instrumentalización o tecnificación corporal?

\section{c) La construcción del proyecto de centro en la escuela $N^{\circ} 267$}

La llegada del EFI a esta escuela acompañó un momento de cambio profundo para la misma, que hasta 2017 fue una escuela común y se transformó en una escuela de tiempo completo en 2018. Esta transformación institucional presentó una oportunidad con relación al formato escolar, trajo consigo innumerables modificaciones que no solo tienen que ver con la extensión del tiempo pedagógico, sino que se abrió la posibilidad de nuevas formas de habitar los tiempos y espacios, el ingreso de nuevos docentes (talleristas) con otros saberes. Todo ello representa un desafío para las maestras y maestros en cuanto a la problematización de su sentido y permite transitar de una mejor manera la escuela y brindar más oportunidades y desafíos para los niños.

Es por eso que el EFI se incorporó a las salas de coordinación, para colaborar con la construcción del proyecto de centro y a la vez sistematizar las discusiones sobre cómo se llevó adelante el cambio para el colectivo docente.

Para el análisis se tomaron como insumos la observación participante de las salas de coordinación. ${ }^{9}$ Si bien estas salas son semanales, el equipo del EFI asistió quincenalmente. Para este trabajo nos centramos en dos categorías de análisis, que son cuerpo y sujeto.

A partir de una de las primeras salas donde se presentó la pregunta sobre "¿qué escuela queremos?", se desarrollaron interesantes discusiones entre los docentes en las cuales aparecieron insumos de análisis que luego fueron compartidos con el colectivo e incorporados en el proyecto de centro. La preocupación por lo corporal, por despertar otro tipo de sensi-

9) Las escuelas de tiempo completo cuentan con una sala de coordinación semanal de dos horas y media. Las mismas deben ser planificadas y gestionadas por el equipo de dirección con la colaboración de los maestros, maestras y demás profesores de la escuela. Allí se abordan temas desde las diferentes dimensiones: organizativo-administrativa, pedagógica, didáctica y comunitaria. 
bilidades, por pensar que a partir del cambio de formato es necesario y urgente otro tipo de propuesta donde la escuela pase por el cuerpo, es lo que nos permitió sumar la mirada de Scharagrodsky (2013), conforme a la siguiente cita:

"Resignificar el cuerpo y ciertas partes del mismo, las emociones y sensaciones, los usos del cuerpo en el espacio y en el tiempo, el cuidado del cuerpo, la sexualidad, el deseo, el placer y los contactos corporales, y resignificar la violencia simbólica, psicológica y física se convierten en acciones prioritarias para cualquier desafío pedagógico y escolar. Aunque en un contexto mucho más complejo y contradictorio, la disyuntiva escolar parece tan vieja como nueva. La cuestión es si el desafío toma "cuerpo" o no lo toma. Quizás valga la pena animarse a construir nuevos sentidos y significados del y sobre el cuerpo, ampliando la voz y la sensibilidad a aquellas corporalidades que han sido sistemáticamente silenciadas, omitidas, sometidas y excluidas". (2013:15)

Es a partir de esta cita y el texto que se comparte que el proyecto coloca como uno de los subtítulos: "El lugar del cuerpo en la escuela" para dar visibilidad a eso que pareciera que la escuela siempre ha olvidado. Pero dar visibilidad al cuerpo no significa que con ello estemos cambiando de mirada hacia él, porque también aparecen los discursos que hacen referencia a que los aprendizajes pasen por el cuerpo para que ayuden a aprender mejor matemáticas, a los procesos de lectoescritura, en fin, la utilización del cuerpo como herramienta. Es difícil hacer una ruptura con algo que viene dado desde la configuración de la escuela tradicional y salir de este aparente ausentismo de lo corporal, aunque este, según Milstein y Mendes (1999), siempre estuvo allí. El cuerpo nunca es tomado como un fin en sí mismo para las prácticas, sino que aparece como medio para algo de mayor significancia, como el intelecto. Pero sin dudas que comenzar a problematizar el lugar del cuerpo en la escuela, preocuparse por repensar distintas prácticas educativas que coloquen al cuerpo como eje de análisis, y dar al arte otro lugar en las prácticas, permite que algo irrumpa en los modelos dominantes que configuran el orden corporal escolar.

Por otro lado, también surgió con énfasis la discusión sobre el sujeto de la educación, la preocupación por mirarlo con la idea de que el niño puede y de que no porque provenga de un contexto desfavorable la escuela no tiene nada para ofrecerle más que el cuidado. La preocupación por destacar el rol de la escuela como un espacio educativo por excelencia pero que a la vez tiene una relación con la enseñanza que no se puede abandonar hizo colocar también la discusión en la tensión entre sujeto carente y sujeto de la posibilidad que plantea Martinis (2006), y la escuela se posicionó con su proyecto de centro en el segundo polo. Aunque se presentan caracterizaciones de los niños de la escuela como sujetos carentes debido a las problemáticas del contexto en el que viven, la posición de los docentes es mirar a los sujetos como "sujetos de posibilidad" (Proyecto de centro de la escuela № 267).

Luego de finalizada la construcción de dicho proyecto, se continuó asistiendo a las salas y se comenzó con la problematización y análisis de un espacio de convivencia diario que sucede a la hora de la entrada y que le otorga identidad a la escuela. 


\section{d) Elaboración de un proyecto de trabajo en el espacio de coordinación \\ docente Liceo $N^{\circ} 42$}

En el Liceo, el EFI se planteó como objetivo potenciar y dinamizar las salas docentes ${ }^{10}$ a través del acompañamiento en el armado de su proyecto institucional. En el proceso los docentes presentaron como problema central la imagen del Liceo, puesto que detectaron que la misma hacía unos años se había venido deteriorando y eso había llevado a que algunos adolescentes del barrio emigraran a liceos más lejanos, lo que generó, a su vez, un descenso importante en la matrícula de este Liceo y llevó sistemáticamente a la pérdida de grupos año a año.

Para abordar este problema, se elaboró un posible camino y se pusieron en discusión algunos elementos teóricos de la planificación estratégica de Aguerrondo (1990).

En este territorio, los avances en el análisis fueron posibles a partir de la implementación de la propuesta de un FODA (Fortalezas, Oportunidades, Debilidades y Amenazas), ${ }^{11}$ en tanto se dio un lugar protagónico al equipo docente para poder identificar colectivamente la situación actual del Liceo. De modo general, el FODA reveló, como debilidad, el mismo problema que habían detectado en cuanto a la imagen del Liceo y también la falta de equipo de dirección efectivo. Como fortalezas ubicaron la infraestructura del Liceo y un equipo de docentes con mucha antigüedad allí y compromiso con la tarea educativa.

En ese contexto se puso en juego la propuesta de pensar en un problema para proyectar líneas de acción concretas. Se partió del disparador de la siguiente cita:

"El perfil concreto del quehacer de la planificación se resume en su capacidad de generar y sostener líneas de acción. Esto ha sido adecuadamente expresado en tres instancias: la efectividad de la planificación depende de tres condiciones; saber hacer, querer hacer y poder hacer. Entonces, si planificar es saber hacer, querer hacer y poder hacer, el común denominador es el HACER, lo que implica que esta perspectiva de planificación/gestión (o planificación/acción) pierde su sentido si no llega a una acción transformadora concreta de la realidad institucional”. (Aguerrondo, 1990:45)

Si bien en esta institución los tiempos del territorio marcaron la dificultad de poder avanzar y profundizar en el análisis desde los ejes del EFI, consideramos que el valor central de este trabajo radica en el lugar político que se toma en las salas. En palabras de Blase (2002:1): "La micropolítica es una dimensión fundamental del cambio escolar en general y más específicamente, un núcleo central de la mayoría de enfoques de la reestructuración". Pensar desde este lugar es colocar a los sujetos de la educación (tanto en la posición alumno como docente) como sujetos políticos y "de posibilidad" (Martinis, 2006), en tanto que "la posibilidad" de producir o no cambios, en este caso en la imagen del Liceo, tiene que ver con la apuesta a generar acciones en colectivo y el convencimiento de que el cambio es posible,

10) Una vez a la semana, en las salas de coordinación son destinadas dos horas a una reunión conjunta del equipo de dirección y los docentes del Liceo para la organización de la propuesta educativa en todas sus dimensiones (organizativo-administrativa, pedagógica, didáctica y comunitaria). Esta reunión puede ser organizada semanalmente o alternando una semana a coordinación general (todos los docentes), una semana a coordinación por nivel, y otra a coordinación por área de conocimiento.

11) Dinámica que se basa en diagnosticar la institución en función de los siguientes cuatro ejes a los que remite la sigla: Fortalezas, Oportunidades, Debilidades y Amenazas, siendo los dos primeros positivos y los dos últimos negativos; el primero y el tercero, internos; y el segundo y el cuarto, externos a la institución. 
impactando en "la posibilidad" de salir de esa "imagen negativa" del Liceo que estaba enlazada con el "sujeto carente". Mediante el proceso de escritura del proyecto de centro se buscó revertirla en parte, y emergieron poco a poco discursos docentes que habilitaron el hacer y sus propias posibilidades para el cambio, de modo de permitir otras formas de configurar a los alumnos de los cuales surgieron algunos rastros de un sujeto "político" y "de posibilidad".

\section{Consideraciones finales}

En esta síntesis del trabajo realizado durante el año 2018 con cuatro instituciones ubicadas en Malvín Norte se dejaron planteados los principales ejes de análisis y reflexión desarrollados con cada uno de los colectivos institucionales. Se intentó dar cuenta de la articulación de los problemas del territorio y los marcos teóricos del EFI en un trabajo de artesano que se fue moldeando y construyendo a lo largo del proceso recorrido junto a los actores de las instituciones.

Es importante destacar cómo las diversas experiencias lograron despertar preguntas teóricas que obligaron a la búsqueda de nuevas discusiones, y estas, a su vez, fueron interpeladas por las problemáticas surgidas en el territorio. En este sentido, es importante mostrar el lugar de los equipos institucionales en la reflexión y análisis de las experiencias seleccionadas a partir de marcos y referencias teóricas que se iban amalgamando, que lograban modificarse y, en cierta medida, tomaban cuerpo por medio de la interpretación de los diversos registros.

Algunas categorías analíticas que atravesaron varias de las experiencias analizadas merecen especial atención. La mayoría de los análisis de los registros de las prácticas educativas aquí relevadas muestra la tensión entre los procesos de rutinización, reproducción, repetición que remiten a la estructura discursiva y la dimensión de acontecimiento que la agujerean y evitan su cierre y saturación, dando lugar a la producción de algo nuevo.

Los análisis desarrollados con diferentes énfasis y modulaciones evidenciaron los modos de inscripción del cuerpo, el saber y el sujeto en la tensión entre la reproducción y el cambio, tratando de escapar a análisis binarios y de no caer en reduccionismos tales como que en todo ritual lo que predomina es la reproducción y en toda práctica abierta, espontánea, lo que prima es el acontecimiento y la producción de algo nuevo. Los análisis y el trabajo de problematización desarrollado a lo largo del año con cada una de las instituciones dan cuenta de la importancia de ver la posibilidad de desestabilización de los rituales, prácticas repetitivas y necesarias en todo acontecer institucional y encontrar en ellas lo disruptivo, desnaturalizar lo evidente. En el mismo sentido, esto permitió salir de la creencia de que las prácticas supuestamente más libres o espontáneas, aquellas que se abrían al espacio para la creación, lo lúdico y artístico per se estaban libradas de la reproducción y la normalización corporal del sujeto y del saber.

\section{Referencias bibliográficas}

Agamben, G. (2003). Infancia e Historia. Buenos Aires: Adriana Hidalgo.

Aguerrondo, I. (1990) El planeamiento educativo como instrumento de cambio. Buenos Aires: Troquel. Alonso, V. (2007). Técnica y educación desde un saber como techné a una tecnología del cuerpo. Educação 
Temática Digital, 8(esp.), 314-332. Campinas: Unicamp. Recuperado de: http://nbn-resolving.de/urn:nbn:de:0168-ssoar-73915 (consultado el 12/10/18).

Behares, L. (2004). Enseñanza-Aprendizaje revisitados. Un análisis de la "fantasía didáctica". En Behares, L.E. (Dir.). Didáctica mínima. Los Acontecimientos del Saber (pp. 11-30). Montevideo: Psicolibros.

- (2007). Enseñar en cuerpo y alma: la teoría de la enseñanza y el saber en la pulsión. Educação Temática Digital, 8(esp.), 1-21. Campinas: Unicamp. Recuperado de: http://143.106.58.55/revista/index.php (consultado el 12/10/18).

(2010). Saber y terror de la enseñanza. Montevideo: Psicolibros.

Blase, J. (2002). Las micropolíticas del cambio educativo. Recuperado de: http://www.ugr.es/ recfpro/rev61ART2.pdf (consultado el 1/12/17).

Chevallard, Y. (1991). La transposición didáctica. Del saber sabio al saber enseñado. Madrid: Aique.

Dogliotti, P. (2007). La imposible enseñanza del cuerpo. En Bordoli, E.; Blezio, C. (Comps.). El borde de lo (in) enseñable. Anotaciones sobre una teoría de la enseñanza (pp. 131-141). Montevideo: FHCE/UdelaR.

Foucault, M. (2015). Vigilar y Castigar. Nacimiento de la Prisión. Buenos Aires: Siglo XXI Editores $\left(2^{\circ}\right.$ de. $6^{\circ}$ reimp.). $1^{\circ}$ ed. francesa 1975 , Gallimard.

(1992). Historia de la sexualidad. Tomo I. La voluntad de saber. Madrid, Siglo XXI Editores.

Martinis, P. (2006). Educación, pobreza e igualdad: del "niño carente" al "sujeto de la educación". En Martinis, P.; Redondo, P. (Comps.). Igualdad y Educación. Escrituras entre (dos) oriilas (pp. 12-31). Buenos Aires: Del Estante Editorial,

Milstein, D.; Mendes, H. (1999). La escuela en el cuerpo. Estudios sobre el orden escolar y la construcción social de los alumnos en escuelas primarias. Buenos Aires: Unigraf.

Pêcheux, M. (1988). O discurso. Estrutura ou Acontecimento. Campinas: Pontes Editores.

Scharagrodsky, P. (2013). El cuerpo en la escuela. En Explora, las ciencias en el mundo contemporáneo. Buenos Aires: Ministerio de Educación, Ciencia y Tecnología.

UdelaR (2010). La extensión en la renovación de la enseñanza: Espacios de Formación Integral. Colección Hacia la Reforma Universitaria. Documento No 10. Rectorado. Montevideo: UdelaR. Recuperado de: http:// www.extension.udelar.edu.uy/wp-content/uploads/2016/12/08_Hacia-la-reforma-universitaria_-la-extensio\%CC\%81n-en-la-renovacio\%CC\%81n-de-la-ensen\%CC\%83anza.pdf (consultado el 25 de abril de 2019). 